\title{
Participación ciudadana y planificación del desarrollo local: caso del cantón Gualaceo
}

Citizen participation and local development planning: case of the Gualaceo canton

\author{
Mercy Alexandra Méndez Pinos. ${ }^{1}$
}

Recibido: 02-02-2021 / Revisado: 11-02-2021 /Aceptado: 02-03-2021/ Publicado: 05-04-2021

\begin{abstract}
.
DOI: https://doi.org/10.33262/concienciadigital.v4i2.1625

Introduction. Citizen participation in Ecuador goes beyond electing rulers, it is a right of citizens to intervene in a leading way in public issues, it is a continuous process of building citizen power. This work aims to analyze the citizen participation processes applied in the Autonomous Decentralized Government of the Gualaceo canton during the period 2011-2019 and how they have been contributing to local development. Objective. To design a methodological proposal for citizen participation in the local development planning process in the case of Gualaceo canton. Methodology. This research has a quantitative and qualitative approach with analytical and purposeful scope. Starting with the data collection technique, the same one that corresponds to primary data such as a survey carried out on citizens over 15 year of age residing in the Gualaceo canton and a semi-structured interview directed to officials linked to citizen participation during the period 2011-2019 and secondary data such as the bibliography review to obtain theories and concepts regarding citizen participation and local development planning, as well as the legal instruments of the Constitution and the application of the mechanisms provided by the CPCCS Council for Citizen Participation and Social Control. Result. As a result of the research, a methodology to promote encourage citizenship is proposed, in order to develop individual and collective human talent, especially in young people to train new leaders who are involved and committed to the local development of the canton; this proposal is subjected to a validation process according to the criteria of experts linked to citizen participation. Conclusion. The suggested methodological proposal for citizen participation has an acceptance of $65.63 \%$ according to the criterion of experts, as they
\end{abstract}

\footnotetext{
1 Universidad Católica de Cuenca, Posgrado, Maestría en Desarrollo Local, mención Planificación, Desarrollo y Ordenamiento Territorial, Cuenca, Ecuador, mercybon@ hotmail.com, https://orcid.org/00000001-5186-1394.
} 
consider that it is pertinent, coherent and viable for any instance of citizen participation of the Gualaceo canton.

Keywords: citizen participation, planning, local development, decentralized autonomous government, Gualaceo canton, Constitution.

\section{Resumen.}

Introducción. La participación ciudadana en el Ecuador va más allá de elegir a los gobernantes, es un derecho de los ciudadanos de intervenir de forma protagónica en temas públicos, es un proceso continuo de construcción del poder ciudadano. Este trabajo tiene el propósito de analizar los procesos de participación ciudadana aplicados en el Gobierno Autónomo Descentralizado del cantón Gualaceo durante el periodo 2011-2019 y cómo han venido contribuyendo al desarrollo local. Objetivo. Diseñar una propuesta metodológica para la participación ciudadana en el proceso de planificación del desarrollo local en el caso del Cantón Gualaceo. Metodología. La presente investigación es de enfoque cuantitativo y cualitativo con alcance analítico y propositivo. Partiendo con la técnica de recolección de datos la misma que corresponde a datos primarios como una encuesta realizada a ciudadanos mayores de 15 años en adelante residentes en el cantón Gualaceo y una entrevista semi estructurada dirigida a funcionarios vinculados a la participación ciudadana durante el periodo 2011-2019 y datos secundarios como la revisión de bibliografía para obtener teorías y conceptos respecto a la participación ciudadana y planificación del desarrollo local, así también los instrumentos legales de la Constitución y la aplicación de los mecanismos dispuestos por el Consejo de Participación Ciudadana y Control Social CPCCS. Resultado. Como resultado de la investigación se propone una metodología de fomento y estímulo a la ciudadanía, con el fin de desarrollar talento humano individual y colectivo, de manera especial en los jóvenes para formar nuevos líderes partícipes y comprometidos en el desarrollo local del cantón; esta propuesta es sometida a un proceso de validación según criterio de expertos vinculados a la participación ciudadana. Conclusión. La propuesta metodológica para la participación ciudadana sugerida, tiene una aceptación del 65,63\% según el criterio de expertos, pues consideran que es pertinente, coherente y viable para cualquier instancia de participación ciudadana del cantón Gualaceo.

Palabras claves: participación ciudadana, planificación, desarrollo local, gobierno autónomo descentralizado, cantón Guacaleo, Constitución.

\section{Introducción.}

El mundo en general y Latinoamérica han vivido muchas transformaciones en distintos ámbitos, de manera especial lo relacionado a la democracia que durante las dictaduras la participación ciudadana siempre estuvo ausente en todo su contexto. Con el retorno a la democracia como una nueva opción de gobierno, la participación ciudadana empieza a 
tomar protagonismo como "democracia representativa" otorgando a los ciudadanos una mayor capacidad de intervención e influencia en la toma de decisiones de carácter público.

La participación sin el término ciudadana carece de impacto pues no se puede entender si no está dentro de un régimen específico como es la democracia, del cual se desprenden conceptos como sociedad civil, tercer sector y participación ciudadana que conjuntamente con la gobernanza son dos esquemas que son parte del desarrollo de una sociedad.

En el Ecuador, la participación ciudadana empieza en la década de los 90 con el movimiento indígena, el cual llevó a cabo grandes paralizaciones en búsqueda de bienestar e igualdad. Moncada (2012) y Carrera (2015) concuerdan que es un proceso en construcción y depende de condiciones políticas, económicas y sociales para obtener buenos resultados.

La participación ciudadana es un tema de interés público, debido a que involucra varios actores sociales que ejercen influencia en el desarrollo de un sector. A partir de la creación de leyes de Participación Ciudadana en el año 2010, en el Ecuador la participación ciudadana y la gestión del desarrollo local son dos proyectos que están contribuyendo en las nuevas tendencias de la administración pública, por lo que es necesario que los gobiernos locales empleen nuevas tácticas de gestión participativa en proyectos a realizarse. En la actualidad es utilizado como herramienta para la gobernanza, es reconocida y aplicada no solo por el gobierno central, sino que también es adoptada por mandato constitucional por los gobiernos autónomos descentralizados GAD’s.

El presente estudio radica en el análisis de los mecanismos aplicados en el GAD del cantón Gualaceo desde la regulación de la participación ciudadana en el Ecuador, establecidos en la Constitución en octubre del 2008 y en la Ley de Participación Ciudadana publicado en abril del 2010, siendo el objetivo general diseñar una propuesta metodológica para la participación ciudadana que intervenga y contribuya en el proceso de planificación del desarrollo local del Cantón Gualaceo.

\section{Teorías sobre la planificación del desarrollo local.}

La planificación convencional es una herramienta que conduce a obtener un objetivo en un determinado tiempo y espacio. Blanes, lo define como "la acción para alcanzar una serie de objetivos previstos de antemano, y haciendo un uso adecuado de los recursos disponibles" (2011, p. 8). Lira, señala que "es un método de intervención para producir un cambio en el curso tendencial de los eventos" (2006, p. 7). Perrotti, lo señala como "el proceso mediante el cual se racionaliza la ejecución de un proyecto nacional de desarrollo, y a partir del cual se identifica una imagen-objetivo" (2014, p. 15).

Las definiciones anteriormente expresadas concuerdan que la planificación es un proceso planeado, meditado y estructurado mediante el cual las personas siguen, con la única razón de alcanzar objetivos basados en una lógica, que conduzca y evidencie un cambio 
sustancial en un determinado tiempo y con los recursos disponibles, existen otras definiciones como la de Dante Ayaviri (2016) que señalan a la planificación como instrumento para el desarrollo.

Por otro lado, el desarrollo es la acción de desarrollar o de desarrollarse, es un proceso que conduce alcanzar el bienestar de una sociedad, Casellas (2014) menciona en su texto que los inicios del desarrollo se dieron durante la revolución francesa, con el cambio de la estructura social, política e industrial que condujeron a profundas transformaciones. Por su lado Rodríguez, considera que el desarrollo "se adapta mediante nuevas formas de organización y de gestión, también mediante nuevas formas de producción al mundo en el que se desenvuelve" (2009, p. 39). Para Figueroa (2012) y Barrera (2018) el desarrollo está ligado a la teoría económica como medida de progreso de las sociedades occidentales en la organización de sus recursos. Los tres autores señalan al desarrollo como sinónimos de evolución y progreso que implica cambio y crecimiento, que sin la participación del ser humano carece de sentido.

Blanes, expone que existen tres paradigmas que han cambiado la forma de manejar la planificación del desarrollo, sin embargo, cada una tiene su propia fundamentación y lógica:

- Planificación estratégica, inicialmente fue incorporada desde la planeación de las estrategias militares, sus principios están en alcanzar el objetivo deseado.

- Planificación integral del desarrollo, tiene una visión global del territorio, se relaciona el proceso de desarrollo con componentes que se requiere para alcanzarlo.

- Planificación participativa, es la participación de los actores sociales a lo largo del proceso de planificación, defiende la tesis de una participación inclusiva que supere los límites de la desigualdad de una sociedad. (2011, p. 11)

En el análisis de experiencias de planificación de los países de América Latina presentan un concepto que abarca nuevas tendencias:

La planificación del desarrollo permite incorporar, en forma secuencial y sistemática, objetivos y metas a largo plazo; estos obedecen a un proceso participativo y estratégico que puede ser motivado e impulsado por el Estado, en otros casos por el sector privado y también desde la población. (Dante Ayaviri et al., 2016, p. 79)

El concepto de desarrollo local aparece en la década de los 70 durante la crisis que sufrieron los países industrializados, como alternativa para mejorar el potencial humano con la introducción de nuevas tecnologías, a fin de superar las formas tradicionales de desarrollo. Para autores como García (2007), Juárez (2013), Gallicchio y Camejo (2005) el desarrollo local implica cambio, crecimiento, pero sobre todo equidad, sostienen que con estos principios se podría obtener un espacio más productivo donde el individuo pueda actuar, elevar su nivel de vida, aportar al desarrollo de su país y afrontar los desafíos de la globalización a largo plazo. 
Para Gallichio (2004)) y Suárez (2006) el desarrollo local es un proceso que requiere la colaboración de actores públicos de distintos niveles (locales, regionales, nacionales e internacionales), también la intervención del sector privado que son los ejes centrales del proceso. Por otro lado Cárdenas señala al desarrollo local como "un proceso que se construye diferenciadamente en cada país según las distintas articulaciones que se producen entre las dimensiones territoriales, la historia, las estructuras y la acción diferenciada de los actores" (2002, p. 61,62). En términos generales, el desarrollo local consiste en identificar y aprovechar los recursos propios de una comunidad, barrio o ciudad que junto con el ser humano como actor local generen nuevos enfoques, oportunidades y desafíos que conduzcan a mejorar una visión hacia el futuro.

\section{Participación ciudadana y democracia.}

La participación ciudadana de manera general es considerada como una organización sin fines de lucro, que brinda su aporte desde la sociedad civil. Carrera (2015) y Sánchez (2009) lo consideran como un derecho que tenemos la ciudadanía en general para incidir de manera individual o colectiva en temas de interés público. Por su lado, Valarezo y Torres (2013) consideran a la participación como un proyecto de colaboración, oportuno y con costos bajos que permite a la ciudadanía vigilar y empoderarse del desarrollo.

Ait Kaci (2015) en la Declaración Universal de Derechos Humanos de las Naciones Unidas, art. 21 señala tres puntos importantes sobre la participación ciudadana, 1. sobre los derechos a participar en el gobierno de cada país, 2. la igualdad de las personas en las funciones públicas de sus países, y 3. la voluntad del pueblo con respecto al voto.

Autores como Camacho (2018), Castillo (2017) y Canto Chac (2008) concuerdan que en América Latina la participación ciudadana está vinculada con la gobernanza a partir de tres puntos importantes como son: la democracia, los derechos y el desarrollo, que junto a las políticas públicas estaría la relación entre el gobierno y la sociedad. Para Guillen, "es un elemento esencial en la construcción y consolidación de las democracias modernas" (2008, p. 134). En consecuencia, la participación ciudadana no solo es la relación entre el estado y la sociedad, es mucho más que eso, se trata de construir estados sostenibles y sustentables, por medio de mecanismos, que generen políticas públicas inclusivas en las que se involucren activamente los ciudadanos.

El propósito de todo proceso participativo es optimizar la democracia, tomando en cuenta siempre la legalidad de las actuaciones públicas y sus instituciones responsables; sin embargo, esta participación es limitada y en algunas ocasiones negativas, debido a que muchas veces son consideradas como un instrumento que vela los intereses únicamente de los políticos con insuficiente poder representativo social o guiados por ciudadanos desinformados y con escasa ética. Una autentica planificación para el desarrollo demanda una participación ciudadana responsable y dinámica, el cual se construye con procesos de educación y capacitación específicamente sobre derechos de los ciudadanos. 


\section{Participación ciudadana y control social en Ecuador.}

La participación ciudadana en el Ecuador empieza a institucionalizarse a partir de la Constitución en 1998 y posteriormente en el 2008 cuando se establece la base legal para promocionar mecanismos y formas para intervenir en lo público, además se constituye un espacio llamado "derechos de participación" con mecanismos de democracia representativa, comunitaria, directa, entre otros. Con esta base legal se establece que la participación ciudadana es un hecho voluntario para tener uso de los derechos establecidos en la ley y en la Constitución.

El Consejo de Participación Ciudadana y Control Social CPCCS, es un organismo que forma parte de la Función de Transparencia y Control Social, considerado como la cuarta función del estado según el orden constitucional, encargado de controlar errores, excesos o arbitrariedades de las instituciones públicas y de las personas que laboran en estas. Las principales normativas referentes a la participación ciudadana se encuentran en la Constitución, en la Ley Orgánica de Participación Ciudadana y Control Social, en el Código Orgánico de Ordenamiento Territorial, Autonomías y Descentralización (COOTAD), Código Orgánico de Planificación y Finanzas Públicas y en la Ley de Transparencia.

Alvarez y Villacreses (2016) señalan que existen instrumentos que favorecen la participación ciudadana como:

- La iniciativa ciudadana: para la aplicación de normas en la implementación de políticas públicas.

- Los buzones ciudadanos: para receptar opiniones, demandas o sugerencias respecto a acciones gubernamentales.

- El monitoreo ciudadano: para dar seguimiento a las políticas específicas.

- La contraloría ciudadana: que permite auditar y evaluar las políticas implementadas.

- La intervención de actores: no vinculados al gobierno en las distintas fases del proceso de implementación de las políticas públicas.

Freire (2016) señala en su documento que el deber más importante del estado es hacer respetar los derechos humanos como garantiza la Constitución, velando por el cumplimiento de la ciudadanía en participar en todas las instancias de la gestión pública de las distintas funciones del estado y niveles del gobierno a través de mecanismos e instancias, así como lo señala el Art. 95 de la Constitución:

Las ciudadanas y ciudadanos, en forma individual y colectiva, participarán de manera protagónica en la toma de decisiones, planificación y gestión de los asuntos públicos, y en el control popular de las instituciones del Estado y la sociedad, y de sus representantes, en un proceso permanente de construcción del poder ciudadano. La participación se orientará por los principios de igualdad, autonomía, deliberación pública, respeto a la diferencia, control popular, 
solidaridad e interculturalidad.

La participación de la ciudadanía en todos los asuntos de interés público es un derecho, que se ejercerá a través de los mecanismos de la democracia representativa, directa y comunitaria. (2008, p. 43)

La Ley Orgánica de Participación Ciudadana (2011) en el Art. 64, establece que en todos los niveles de gobierno existirán instancias de participación con el fin de elaborar planes, políticas, agendas de desarrollo, presupuestos participativos, rendición de cuentas y por sobre todo promover la formación ciudadana. Las instancias donde se presenta participación ciudadana en todos los niveles de gobierno son: la Rendición de Cuentas, el Presupuesto Participativo y los Planes de Desarrollo y Ordenamiento Territorial (PDOT) y también en otros procesos como:

Asambleas: Espacio donde las organizaciones de ciudadanas y ciudadanas son convocadas para dialogar junto a autoridades de gobiernos locales temas de interés común relacionados a la gestión pública de la localidad.

Silla Vacía: En este mecanismo se señala que el espacio vacío será ocupado por uno o por varios representantes de la ciudadanía, dependiendo del o los temas a tratar, a fin de participar en el debate y posterior en la toma de decisiones. La convocatoria se la hace con anticipación, y el o los ciudadanos que intervengan en la sesión debe estar acreditados y sujetarse a la normativa pertinente de los GAD’s.

Audiencia Pública: Este mecanismo tiene como objetivo atender alguna petición o manifestación ciudadana relacionados con la gestión pública, a fin de discutir problemas que afecten a los intereses de los ciudadanos.

Veedurías: Es un mecanismo de control social, mediante el cual los representantes de la ciudadanía, vigilan, fiscalizan y controlan la administración y la gestión de una institución que maneje o desarrolle actividades públicas.

Zicardi (1998) señala que, al participar en estos mecanismos, el ciudadano indirectamente está trabajando para el desarrollo de su sector y que es el mejor espacio para generar una relación entre el gobierno y la ciudadanía.

\section{Caso de estudio: Cantón Gualaceo}

Gualaceo, es el segundo cantón con mayor población de la provincia del Azuay, ubicado a $35 \mathrm{~km}$ de la capital provincial Cuenca, se le denomina "Jardín del Azuay" al estar bañado por los ríos Santa Bárbara y Guaymincay. Limita al Norte con los cantones Paute y Guachapala, al Sur con los cantones Chordeleg y Sigsig, al Este con los cantones El Pan y Limón Indanza y al Oeste con el cantón Cuenca.

La superficie que alcanza es de $345,48 \mathrm{~km} 2$, la altitud va entre los 2.100 a 4.000 metros sobre el nivel del mar, la temperatura varía entre $\operatorname{los} 7^{\circ} \mathrm{Ca} 18^{\circ} \mathrm{C}$, cuenta con una población 
de 44.887 habitantes según el censo realizado en el año 2010 y con una proyección de 49.104 habitantes al año 2020. En la actualidad está conformado por ocho parroquias rurales: Mariano Moreno, Daniel Córdova Toral, Luis Cordero Vega, Remigio Crespo Toral, Jadán, Zhidmad, San Juan y Simón Bolívar más la parroquia urbana del mismo nombre. Las actividades económicas más importantes son: la agricultura, la artesanía y el turismo.

La regulación de la participación ciudadana en el cantón Gualaceo se da inicio con la resolución de dos reglamentos. El primero se constituye el 11 de mayo del 2011, amparándose en los Art. 61, 85, 95 y 100 de la Constitución del Ecuador y en los artículos 302, 303 del Código Orgánico de Organización Territorial Autonomía y Descentralización COOTAD, el consejo cantonal decide disponer del "Reglamento que norma la participación ciudadana en el nivel de gobierno local de Gualaceo" este reglamento trata sobre la conformación de los Sistemas de Participación Ciudadana y la Silla Vacía; el segundo se constituye el 03 de agosto del 2011, de igual manera amparándose en los Art. 264, 295, 297 del COOTAD, en el Art. 28 del Código Orgánico de Planificación y Finanzas Pública, y en el Art. 66 de la Ley Orgánica de Participación Ciudadana, el consejo cantonal resuelve el "Reglamento para la Constitución y Organización del Consejo Cantonal de Planificación del Gobierno Autónomo Descentralizado de Gualaceo" donde se establece los integrantes para este consejo como son el presidente, vicepresidente y secretario.

\section{Metodología.}

La metodología aplicada en la presente investigación es de enfoque cuantitativo y cualitativo con alcance analítico y propositivo. Partiendo con la técnica de recolección de datos la misma que corresponde a:

Datos primarios: obtenidos de una encuesta realizada a ciudadanos mayores de 15 años en adelante residentes en el cantón y una entrevista semi estructurada dirigida a funcionarios vinculados a la participación ciudadana durante el periodo 2011-2019.

Datos secundarios: por medio de la revisión de bibliografía relevante para obtener teorías relacionadas a la participación ciudadana, planificación, el desarrollo local entre otros.

Universo de estudio: está compuesto de la siguiente manera:

- Delimitación territorial: cantón Gualaceo, que contempla el área urbana y expansión urbana.

- Muestra de población: se obtuvo por medio del siguiente calculo:

$\mathrm{N}=$ Universo Poblacional

$\mathrm{Z}=$ nivel de confianza

$\mathrm{P}=$ probabilidad de éxito

$\mathrm{q}=$ probabilidad de fracasopto 
$\mathrm{d}=$ margen de error

$\mathrm{n}=$ tamaño de la muestra calculado

$$
\begin{aligned}
& n=\frac{N \times Z^{2} \times p \times q}{d^{2} \times(N-1)+Z^{2} \times p \times q} \\
& n=\frac{(9610) \times(1,645)^{2} \times 0,50 \times 0,50}{(0,10)^{2} \times(9609)+(1,645)^{2} \times 0,50 \times 0,50} \\
& n=67,18 \approx 68 \text { muestras }
\end{aligned}
$$

\section{Resultados.}

De acuerdo a las respuestas recibidas de las encuestas realizadas a ciudadanos mayores de 15 años en adelante residentes en el cantón Gualaceo, se puede confirmar que la ciudadanía tiene una visión distinta a la que presenta el Consejo de Participación Ciudadana y Control Social CPCCS; según las respuestas, el 41,70\% de los ciudadanos consideran que la participación ciudadana tiene más relación a participación política y electoral (Figura 1); el 75\% confían más en un líder comunitario que en una autoridad (Figura 2); el 66,70\% dicen haber escuchado sobre las asambleas ciudadanas (Figura 3); el 50\% dice no haber participado en alguna actividad dentro de la comunidad, barrio o cantón, pero dicen tener interés en participar en algún momento (Figura 4); el 40\% indican haber tenido una experiencia nada satisfactoria cuando han participado (Figuras 5); el $30 \%$ dicen que no participan porque no confían en las autoridades (Figura 6); el 66,70\% señalan que les motiva a participar cuando tienen que resolver problemas comunitarios (Figuras 7); el 33,30\% conoce algo sobre participación ciudadana porque lo han visto en una página de internet o en redes sociales (Figura 8); el 50\% les interesaría participar en formación ciudadana (Figura 9).

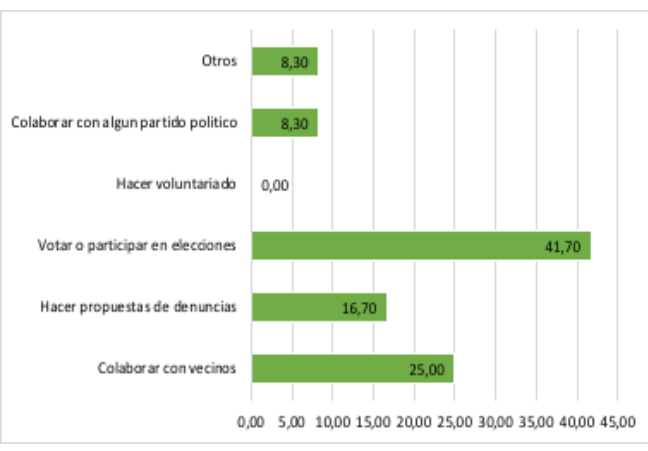

Gráfico 1. Significado de P.C.

Fuente: Elaboración propia

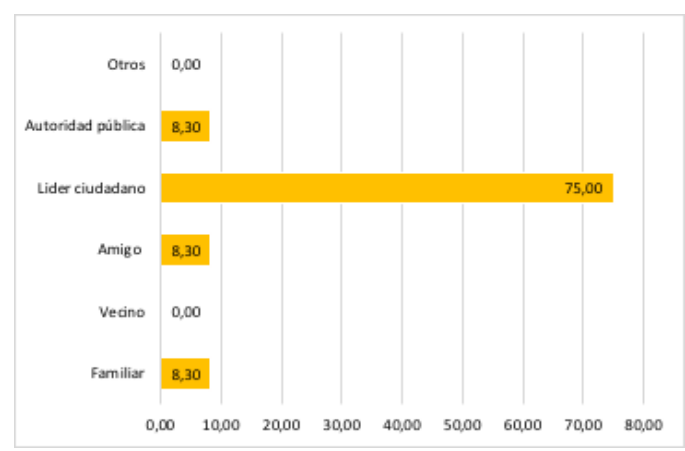

Gráfico 2. Persona que le genera más confianza con temas de P.C.

Fuente: Elaboración propia 


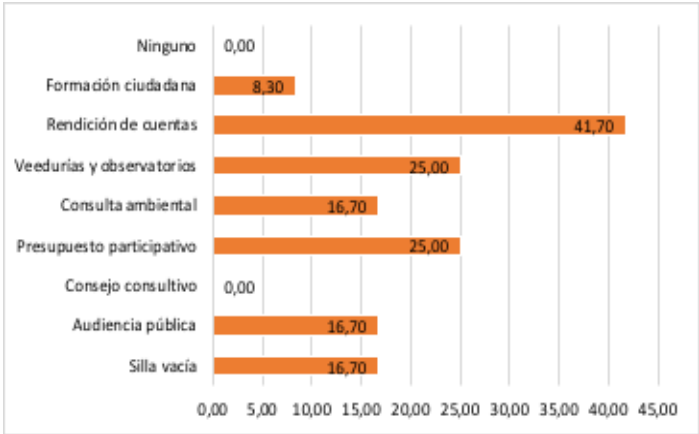

Gráfico 3. Mecanismos de P.C. que ha oído hablar.

Fuente: Elaboración propia

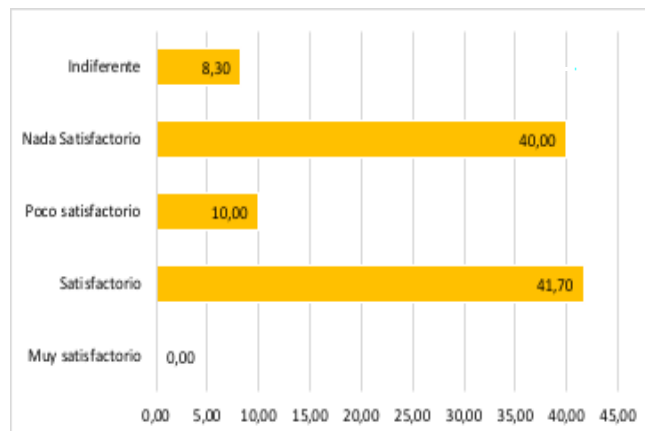

Gráfico 5. Experiencia de P.C.

Fuente: Elaboración propia

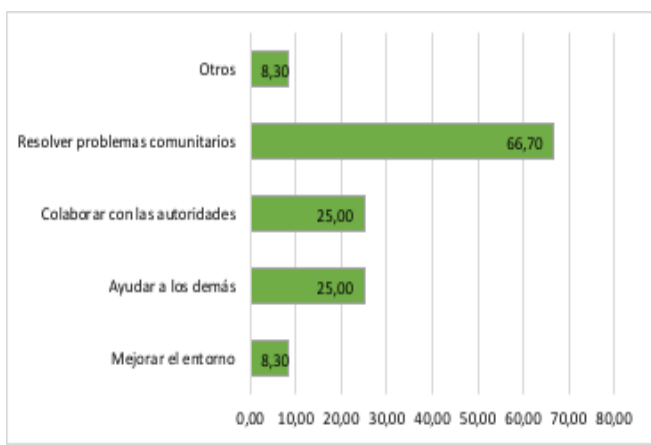

Gráfico 7. Opciones que le motivan a participar. Fuente: Elaboración propia

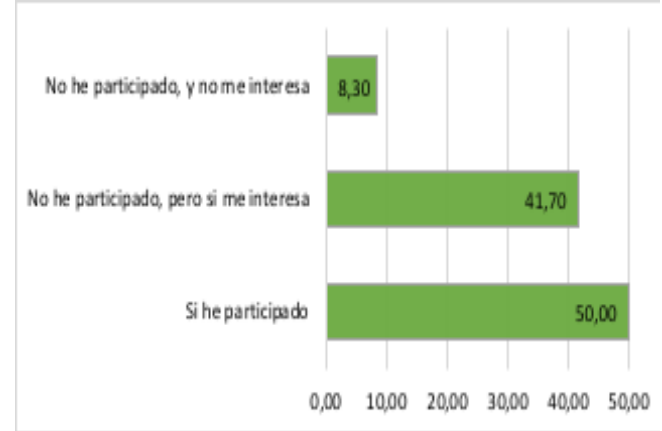

Gráfico 4. Participación Ciudadana dentro de la comunidad, barrio o cantón.

Fuente: Elaboración propia

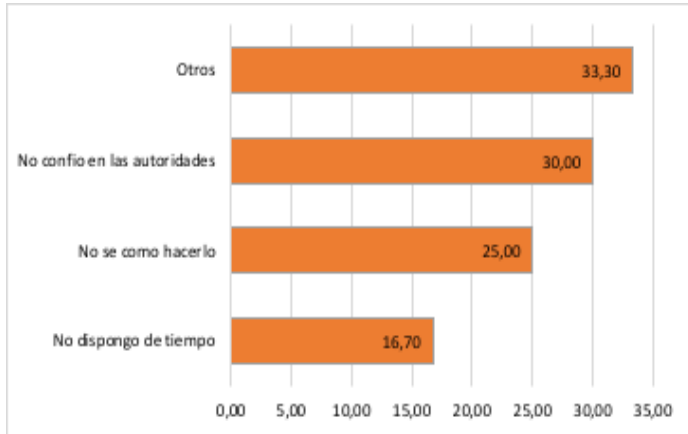

Gráfico 6. Motivos por qué no participa.

Fuente: Elaboración propia

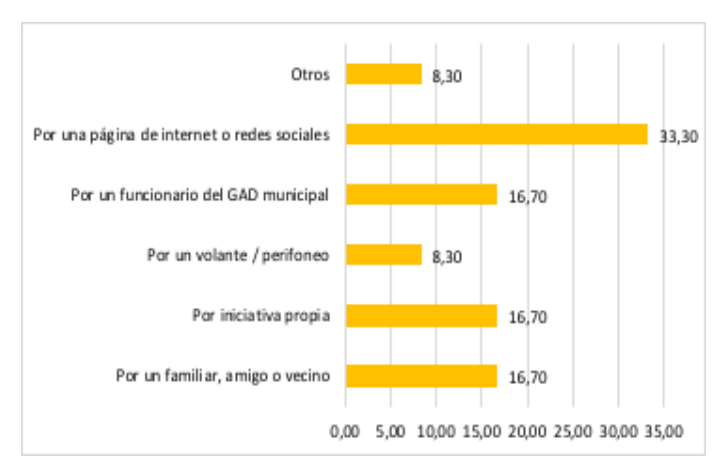

Gráfico 8. Medio por donde se enteró sobre la P.C. Fuente: Elaboración propia

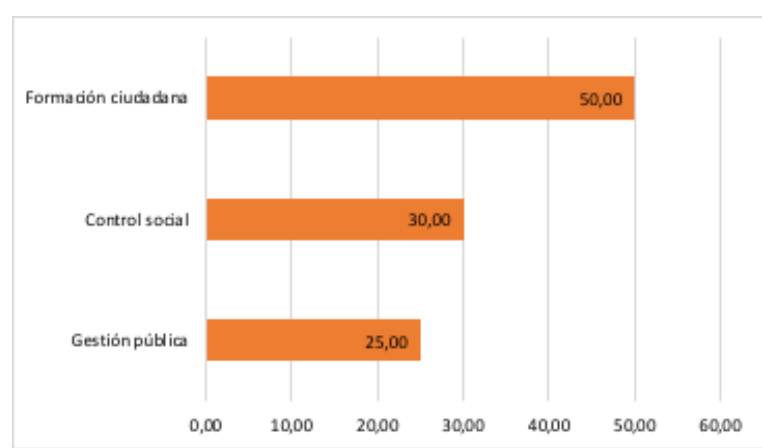

Gráfico 9. Asuntos en los que le interesaría a participar Fuente: Elaboración propia 
De las entrevistas realizadas a funcionarios que ejercieron el cargo durante el periodo de análisis indican que el GAD del cantón Gualaceo si cuenta con Sistemas de Participación Ciudadana desde que se establecieron los reglamentos en el año 2011, las frecuencias de reuniones han ido variando, en el periodo 2011-2014, iniciaron de manera semestral mientras que en el periodo 2014 al 2019 se realizaban de manera anual; los mecanismos de participación ciudadana aplicados durante el periodo 2011-2014 fueron las mesas ciudadanas mientras que en el periodo 2014-2019 fueron las asambleas; el GAD del cantón Gualaceo no cuenta ordenanza para presupuesto participativo, según los funcionarios lo realizaba de acuerdo las guías dispuestas por el Consejo de Participación Ciudadana y Control Social CPCCS. Al contar con un Reglamento para la constitución y organización del consejo cantonal de planificación, el GAD del cantón Gualaceo si ha elegido representantes en los dos periodos, también ha realizado talleres anuales como parte de la formación ciudadana. La rendición de cuentas se ha dado a conocer a través de asambleas en ambos periodos. Los funcionarios a criterio general consideran que los talleres de la formación ciudadana se deben dar más seguido y también se debe implementar talleres específicamente para formación de nuevos líderes.

\section{Discusión}

De los resultados obtenidos en la aplicación de instrumentos en el cantón Gualaceo, se evidencia un desconocimiento de la ciudadanía sobre temas de participación ciudadana, debido a la poca formación que ha tenido la ciudadanía en los últimos años, según los funcionarios indican que podría tratarse también de un problema de tipo cultural, pues señalan que si realizan invitaciones a participar y los ciudadanos no acuden o simplemente no le dan demasiada importancia a este tema.

Por lo consiguiente, resulta indispensable realizar una propuesta metodológica para la participación ciudadana que se adapte al proceso de planificación del desarrollo local del cantón Gualaceo, que sea proactiva, dinámica que conduzca al ciudadano a empoderarse de los asuntos públicos del cantón.

\section{Propuesta.}

La propuesta que se sugiere se fundamenta en el modelo de la Secretaría de Participación y Control Social del Ecuador, la misma que está amparada en el art. 85 de la Constitución que garantiza la participación de la ciudadanía en general en la formulación, ejecución, evaluación y control de las políticas y servicios públicos a fin de convertir estas normas jurídicas en práctica, este modelo es aplicable en todos los niveles de gobierno.

Esta propuesta metodológica es de fomento y estímulo a la ciudadanía con el fin de desarrollar talento humano individual y colectivo de manera especial en los jóvenes para formar nuevos líderes partícipes y comprometidos en el desarrollo local del cantón y de esta manera generar una cultura política amparada en la Constitución. 


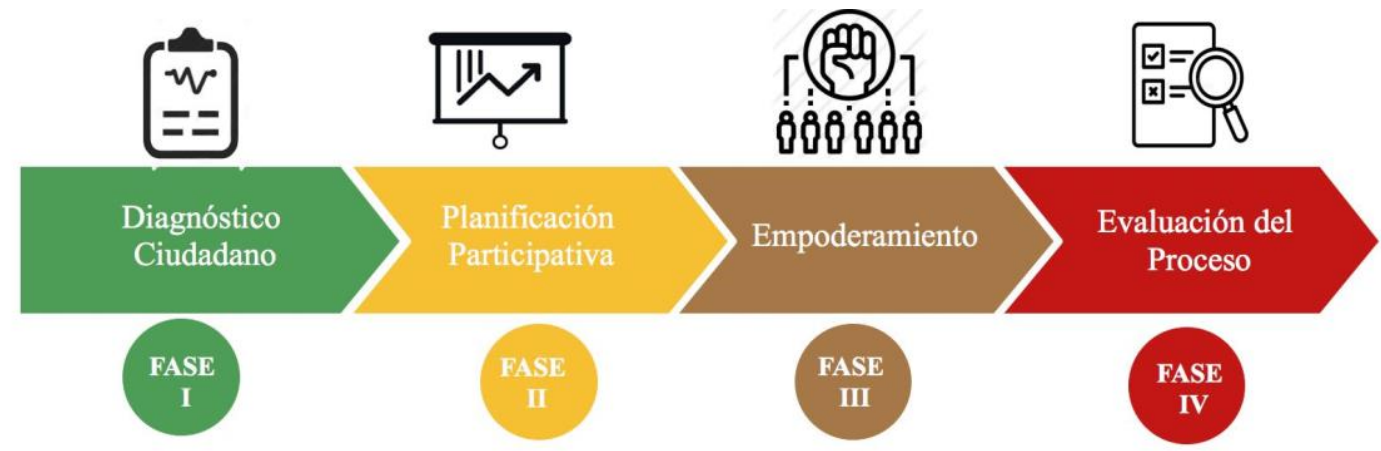

Gráfico 10. Esquema de la propuesta metodológica de participación ciudadana.

Fuente: Elaboración propia

Fase I: Diagnóstico Ciudadano

Parte desde el acercamiento al ciudadano, para conocer los intereses particulares o colectivos, a su vez identificar las organizaciones sociales que se encuentran trabajando por la localidad. Para cumplir con esta fase se debe seguir los siguientes puntos:

- Acercamiento ciudadano.

- Identificación del problema central, línea base del problema y análisis de contexto.

- Actores involucrados.

- Análisis de causa - efecto del problema identificado.

- Planteamiento de objetivos.

Fase II: Planificación Participativa

Partiendo del Diagnóstico Ciudadano donde se identificó la problemática, con apoyo técnico se estructura un plan con respuestas a esas necesidades, encontrando soluciones desde sus propias habilidades. En la planificación participativa se estructuran los proyectos específicos que se pretende desarrollar en la siguiente fase del proceso. En esta fase se inicia con los primeros talleres para elaborar los siguientes puntos:

- Conocimiento de leyes, ordenanzas, convenios, de participación ciudadana del GAD del cantón Gualaceo.

- Elaboración de agenda de incidencia política.

- Plan estratégico.

- Plan operativo.

Fase III: Empoderamiento

En esta fase se reconoce y fortalece las aptitudes individuales y colectivas de los ciudadanos que participan en la formación y capacitación; para cumplimiento de esta fase se tiene que seguir los siguientes puntos: 
- Identidad y liderazgo: el proceso de formación se orienta hacia la "sensibilización ciudadana" en el conocimiento de sus derechos y desarrollo de competencias.

- Promoción de la participación ciudadana: por medio de actividades culturales con temas que promocionen la participación ciudadana, donde los jóvenes puedan participar y paulatinamente vayan integrándose a estos nuevos procesos, se trata de formar nuevos actores sociales.

- Formación y capacitación progresiva: dirigida a miembros de asambleas, organizaciones ciudadanas, veedores y ciudadanía en general con programas sobre derechos de participación y poder ciudadano.

- Normativa jurídica de participación ciudadana: es indispensable que la ciudadanía, y los distintos grupos sociales conozcan, se capaciten e implementen las formas y mecanismos de participación y control social a realidad territorial y local.

- Acompañamiento: después de la formación y capacitación es indispensable que los técnicos acompañen y potencien lo impartido a fin de adquirir sostenibilidad en el proceso.

Fase IV: Evaluación del Proceso

Durante el acompañamiento, se identifican las "Buenas Prácticas", tales como: acciones inmediatas, intercambio de conocimientos, impactos positivos, innovación, etc. De esas buenas prácticas identificadas se extraen los aprendizajes y lecciones obtenidos para posteriormente incorporarlos como modelo. En esta fase de evaluación, se consolidan aquellos elementos que garantizan la sostenibilidad del proyecto.

\section{Validación.}

Para la validación de la propuesta metodológica se evalúa por medio de criterio de expertos, para este caso se receptó la opinión de 4 profesionales vinculados a procesos participación ciudadana en GAD’s cantonales de la provincia del Azuay. El análisis se realiza por medio de una matriz que abarca las fases del proceso, los programas, la evaluación y las observaciones o sugerencias de la propuesta. Los criterios que se aplicaron para cada fase son: la pertinencia, la coherencia y la viabilidad, la forma como se evalúa cada fase: cumple totalmente ( 2 puntos), cumple parcialmente (1 punto) y no cumple ( 0 puntos), se considera propuesta aprobada a partir del $50 \%$ más 1 de los criterios de los expertos. Lo resultados obtenidos de la matriz para evaluación de la propuesta metodológica, son los siguientes:

- Fase I: Diagnostico Ciudadano, esta fase los expertos lo aprueban con el 62,50\%.

- Fase II: Planificación Participativa, esta fase los expertos lo aprueban con el 75\%.

- Fase III: Empoderamiento, esta fase los expertos lo aprueban con el 75\%.

- Fase IV: Evaluación del Proceso, se aprueba con el 50\%. 
Alcanzando un promedio final del 65,63\%, con este resultado se puede indicar que el instrumento queda validado y aceptado para su aplicación según el criterio de expertos.

De las observaciones emitidas, los expertos concuerdan que la propuesta es:

- Pertinente: consideran que es adecuada y oportuna para cualquier instancia de participación ciudadana.

- Coherente: está diseñada acorde a las necesidades que mantiene el GAD del cantón Gualaceo respecto a la participación ciudadana.

- Viable: es aplicable al proceso en términos de recursos y tiempo.

\section{Conclusiones.}

- La Constitución del Ecuador considera a la participación ciudadana como un derecho de la ciudadanía en participar ya sea de forma individual o colectiva en temas de interés público. La Secretaría de Participación y Control Social del Ecuador ha proporcionado guías para promover la participación ciudadana en todos los GAD’s, siendo estas guías la base para ejercer la participación ciudadana conforme lo establece la Ley.

- De los resultados obtenidos de la aplicación de instrumentos en el cantón Gualaceo, los encuestados consideran que la participación ciudadana es la participación que ejercemos los ciudadanos a través del voto, en ese sentido se puede señalar el poco conocimiento que tienen respecto a estos temas, según los funcionarios entrevistados señalan que se debe a la escasa formación ciudadana y también al poco interés que tienen respecto a este tema.

- Debido a esta situación que se presenta en el cantón Gualaceo, es fundamental disponer de una propuesta metodológica para la participación ciudadana que intervenga en el proceso de planificación del desarrollo local del Cantón Gualaceo que motive a la ciudadanía a involucrarse en temas públicos.

- La propuesta metodológica que se sugiere tiene una aceptación adecuada para su aplicación, es una propuesta de fomento y estímulo de la ciudadanía con el fin de desarrollar aptitudes individuales y colectivas de manera especial en los jóvenes para formar nuevos líderes activos y comprometidos en el desarrollo local del cantón Gualaceo.

\section{Referencias Bibliográficas.}

Barrera, Y. J. (2018). La teoría del desarrollo y su influencia en América Latina. Número $1,22-46$.

Blanes, J. P. (2011). Procesos De Desarrollo Y Planificación De Intervenciones.

Camacho Pérez, J. A. (2018). La importancia de la participación ciudadana en las relaciones intergubernamentales: un análisis desde el enfoque de la gobernanza. 
Encrucijada, Revista Electrónica Del Centro de Estudios En Administración Pública, 29, 20.

Canto Chac, M. (2008). Gobernanza y participación ciudadana en las políticas públicas frente al reto del desarrollo. Política y Cultura, 30, 9-37.

Cárdenas, N. (2002). El desarrollo local. 53-76.

Carrera, F. A. (2015). La Participación Ciudadana y Control Social en Ecuador. Uniandes Episteme, 2(1), 19.

Casellas, A. (2014). Desarrollo local y territorio Del crecimiento indiscriminado. Diputació de Barcelona, Elementos(5), 27.

Castillo Cubillos, M. (2017). El papel de la participación ciudadana en las políticas públicas urbanas, bajo el actual escenario de la gobernanza. Revista CS, 157-180.

Constitución de la Republica del Ecuador. (2008). Publicada en el Registro Oficial 449 de 20 de octubre de 2008. Incluye Reformas, 1-136.

Dante Ayaviri, N., Zurita Vaca, M., \& Fierro López, P. (2016). La planificación del desarrollo en América Latina. Un análisis comparativo de Ecuador y Bolivia. In Carta ecnómica regional (Vol. 28, Issue 117).

Figueroa Burdiles, N. (2012). El desarrollo y las políticas públicas. Polis (Santiago), 11(33), 375-392.

Freire, P. (2016). Secretaría Técnica de Participación Ciudadana y Control Social.

Gallicchio, E. (2004). Ponencia presentada en el Seminario "Desarrollo con inclusión y equidad: sus implicancias desde lo Local”, realizado por SEHAS en la ciudad de Córdoba (Argentina), en mayo de 2004. 80, 1-24.

Gallicchio, E., \& Camejo, A. (2005). Desarrollo local y descentralización en América Latina.

Guillen, A. (2008). La participacion ciudadana en el contexto de desarrollo sustentable. Innovaciones de Negocios, 5(1), 131-146.

Juárez Alonso, G. (2013). Revisión del concepto de desarrollo local desde una perspectiva territorial. Líder: Revista Labor Interdisciplinaria de Desarrollo Regional, 23(23), 9-28

Moncada, A. (2012). Voces ciudadanas.

Perrotti, D. E. (2014). Gestión pública. 
Rodríguez J. (2009). Local Development Processes From a European Perspective: Genesis and Transformation Os Processos De Desenvolvimento Local Desde Uma Perspectiva Européia : Genesis E Transformação. 12(24), 37-55.

Sánchez Ramos, M. (2009). La participación ciudadana en la esfera de lo público. Espacios Públicos, 25, 85-102.

Suárez, M. (2006). Universidad y Desarrollo Local en Latinoamérica. Universidad y Desarrollo Local En Latinoamérica., June, 195-211.

Valarezo, G. R. (2013). El desarrollo local en el Ecuador: historia, actores y métodos. In Ediciones Abya-Yala.

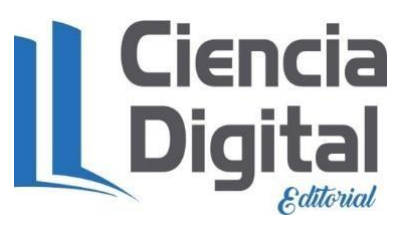




\section{PARA CITAR EL ARTÍCULO INDEXADO.}

Méndez Pinos, M. A. (2021). Participación ciudadana y planificación del desarrollo local: caso del cantón Gualaceo. ConcienciaDigital, 4(2), 22-38.

https://doi.org/10.33262/concienciadigital.v4i2.1625

\section{\Ciencia}

El artículo que se publica es de exclusiva responsabilidad de los autores y no necesariamente reflejan el pensamiento de la Revista Conciencia Digital.

El artículo queda en propiedad de la revista y, por tanto, su publicación parcial y/o total en otro medio tiene que ser autorizado por el director de la Revista

\section{Conciencia Digital.}

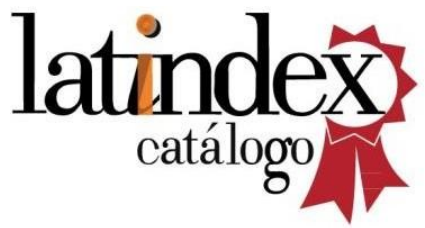

\section{Conciencia}

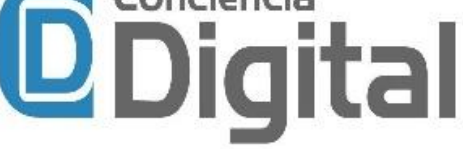

